\title{
A spectrum model for mood disorders: a useful approach in clinical psychiatry in search of an assessment methodology
}

\author{
GIOVANNI B. CASSANO e STEFANO PINI
}

\section{INTRODUCTION}

Psychiatric classification has been a subject of increasing interest over the past two decades. Much progress has been made in diagnostic reliability with the use of relatively uncomplicated prototypes. The complexity of clinical reality is faced in these systems by essentially using two strategies. One is the use of polythetic diagnostic criteria. The other is the possibility of making simultaneously multiple diagnoses in the same individual. However, there remains a serious gap between clinical reality and the options provided by the official nosologies (DSM-IV and ICD-10) which do not fully correspond to the presentations of psychopathology in both community and clinical settings leading to an incomplete characterization of most patients (Cassano et al., 1999).

Such a discrepancy is strikingly evident within the realm of mood disorders, as particularly regards bipolar disorder; although many different terms have been used in the literature to describe subthreshold mania, including soft, mild, subtle, incomplete, partial, atypical and others, these forms of bipolar disorder still are overlooked both in clinical settings and epidemiological studies (Cassano et al., 1983, Cassano \& Savino, 1993, 1997; Cassano et al., 1999).

In this paper we use the term 'subthreshold depressive spectrum' and 'subthreshold bipolar spectrum' to describe those individuals who show features of depression or of bipolar disorder, but whose number of symptoms is not sufficient or are not severe enough to fulfil the diagnostic criteria of DSM-IV or IVCD-10 for depression or bipolar disorder. In addition, the term 'bipolar spectrum' may also be applied to those conditions in which there are atypical symptoms not found in the

Indirizzo per la corrispondenza: Professor G.B. Cassano, Department of Psychiatry, Neurobiology, Pharmacology and Biotechnology, University of Pisa, via Roma 67, 56100 Pisa.

Fax +39-050-21.581 criteria sections of DSM-IV or ICD-10 but indicative of a bipolar diathesis. This paper argues that a systematic study not only of the core symptoms of depression or mania, but also of the associated aura of subthreshold and atypical, manifestations, the importance of which is usually underestimated, may be relevant to diagnosis and have important clinical and therapeutical implications.

The term spectrum has been used to describe relationships among clusters of symptoms and to place a group of defined syndromes in relation to one another (Kety, 1987; Akiskal et al., 1980; Akiskal, 1983; Akiskal \& Pinto, 1999; Hollander \& Wong, 1995; Frank et al., 1998). We instead use the term to refer to broad areas of psychopathological phenomena relating to a single mental disorder, including a) core, atypical, subclinical symptoms of the primary axis I disorder, b) signs, isolated symptoms, symptom clusters and behavioral patterns related to the core symptoms that may be prodromal, may represent precursors of a not yet fully expressed condition, or may be sequel of a previously experienced disorder, and c) temperamental or personality traits (Cassano et al., 1997).

Clinicians often face a complex array of co-occurring, isolated or clustered symptoms that may be associated with one or more axis I or axis II conditions, giving rise to complex clinical situations. The DSM-IV approach tends to trivialize such co-occurring symptoms as background noise interfering with the primary task of matching symptoms to diagnostic criteria (Cassano et al., 1997). Conversely, there is evidence that failure to recognize such symptoms may have clinical and therapeutical implications.

The objective of the spectrum model is to make manifest and give clinical significance to low-severity and isolated symptoms either occurring singly or presenting simultaneously with a major disorder. Practicing psychiatrists need subtle diagnostic tools to detect hidden forms of psychopathology which may be clinically relevant. Less classical unipolar or bipolar symptoms may be obscured by concomitant fragments of other mental disorders, such as, for example, an anxiety disorder or 
substance abuse. In such instances, the underlying mood dys-regulation can be identified by assessing isolated mood signs and symptoms and, in absence of that, by recognizing the course characteristics. Parallel, a substantial proportion of those forms of depression previously labeled as 'masked', 'atypical', 'characterologic', actually appear to be associated with the presence of concomitant anxiety conditions often co-occurring at subsyndromal level or manifesting as enduring underlying traits (Akiskal, 1983; Winokur, 1974; Judd \& Akiskal, 2000).

Although our spectrum model in no way rejects the DSM or ICD affective categories, we argue that the process of identification and diagnosis of such a broad range of features symptoms relevant to a mood disorder can be improved by the introduction of a refined assessment procedure aimed to facilitate the assessment of vulnerability to recurrence or to emergence of more severe forms of psychopathology.

\section{THE SUBTHRESHOLD DEPRESSIVE SPECTRUM: EPIDEMIOLOGICAL EVIDENCES AND CLINICAL IMPACT}

It is not surprising that, much more effort has been devoted to study the various epidemiological and clinical manifestations of subthreshold depression than it was done for subthreshold bipolar spectrum (Schotte \& Cooper, 1999). Perhaps this happened in connection with the common assumption that the depressive spectrum has higher prevalence and greater impact on individual's functioning than subthreshold manifestations of mania may have. Mood symptoms that fall below the threshold criteria for depression have been found to have considerable prevalence rates in general population, as well as in general health care sector and in psychiatric settings. For instance, Judd et al. (1994) using the ECA database described a potential clinical condition represented by 'any two or more simultaneous symptoms of depression, associated with evidence of social dysfunction, occurring in individuals who do not meet criteria for minor depression, major depression and/or dysthymia'. This condition has been defined 'subsyndromal symptomatic depression' and had a prevalence of $11.8 \%$ during a 1 -year period. The authors found that, compared to subjects with no depressive disorder or symptoms, 'subsyndromal symptomatic depression' was associated with insomnia, fatigue, recurrent thoughts of death and more disability in different areas of functioning. A relevant proportion of these subjects experienced depressive symptoms as prodromal to the onset of a major or a minor depressive episode as well as residual to resolving episodes. These findings were consistent with the observation that subsyndromal depressive symptoms might be associated either with an history of past depressive episodes or with a relevant risk factor for future episodes.

Kessler (1997) found that the prevalence of minor depression in the general population ranged between $7 \%$ and $10 \%$ and that several clinical correlates of minor and major depression were similar. Similarly, Sullivan et al. (1998) identified 6 classes of subjects from the NCS, who endorsed different depressive conditions spanning from most severe to mild conditions. The overall prevalence of subthreshold forms of depression was $19 \%$. These conditions, which were hypothesized to be quantitative but not qualitative different from major depression, were associated with social morbidity and an increased risk for first-onset major depression.

Similarly, Angst \& Merikangas (1997) found that the prevalence of lifetime recurrent brief depression and minor depression were $9.2 \%$ and $6.2 \%$ in a sample ascertained from non-psychiatric settings. These subthreshold forms of depression were found to be a risk factor for developing a major disorder and viceversa.

Olfson et al. (1996) recruited a large outpatient primary care sample (1001 subjects), in which subthreshold symptomatology was defined for depression, panic disorder, OCD and substance abuse. A total of $30.1 \%$ of the subjects met the criteria for subthreshold symptoms, whereas 38.9\% met the criteria for an Axis I mental disorder. The criteria for depressive symptoms were met by $9.1 \%$ of the sample. Social impairment was significantly higher in subjects with subthreshold depression than in those without any depressive symptom. Furthermore, one third of subjects with subthreshold depression met the full criteria for another mental disorder, in particular drug or abuse dependence.

Rapaport \& Judd (1998) recruited 15 subjects with subthreshold (two or more symptoms of depression excluding the DSM-IV A criteria) or minor depression (feeling sad or blue or anhedonic' plus at least one other DSM-IV depressive symptom) with no other comorbid psychiatric disorder, from a general practice sample and from the community. They quantified functional impairment and depressive symptomatology in these subjects before and after 8 weeks of pharmacological treatment. Subjects with a current or past history of major depression in the last year were excluded from the sample. When confronted with a general population sample without depressive symptomatology, subjects with minor and subthreshold depression showed lower scores for physical role functioning, emotional role, energy/fatigue, 
emotional well being and social functioning. After a trial with fluvoxamine, depressive symptomatology decreased in minor depressives as well as in subjects with subthreshold depressives symptoms and approximately $30 \%$ of the patients had complete resolution of all depressive symptoms.

\section{THE BIPOLAR SPECTRUM: THE NEED TO BETTER DELINEATE ITS PROFILE AND EPIDEMIOLOGY}

Although there is large consensus that the DSM-IV criteria for a manic episode are limited, perhaps in connection with the stigma associated with a diagnosis of manic-depressive illness, less attention has been given to subthreshold mania. Akiskal et al. $(1980 ; 1983)$ identified a range of expressions of mood disorders he comprised under the label of 'subaffective disorders'. Under this heading he subsumed dysthymia, cyclothymia, and bipolar II disorder; he further noted that in many cases, these conditions are misdiagnosed as 'borderline personality disorder'.

Angst \& Merikangas (1997), in the longitudinal $\mathrm{Zu}$ rich cohort study comprising 591 young subjects from the Community, found high cumulative prevalence rates of hypomania/mania (5.5\%) and brief hypomania (2.3\%), with both categories showing comparable clinical validity. This study suggested that recurrent brief hypomania belongs to the bipolar spectrum.

More recently, Akiskal \& Pinto (1999) highlighting the importance to reveal the presence of a bipolar diathesis as the least common denominator of different phenomenological presentations of depression, described three patterns of bipolar spectrum, namely depression with protracted hypomania, cyclothymic depressions and bipolarity masked/unmasked by stimulant abuse, which represent intermediary subtypes between bipolar types I, II, III and IV, this latter being proposed as hyperthymic depression. All these forms may represent relatively frequent unrecognized or misdiagnosed prototypes of bipolar spectrum. However, these descriptions of bipolar spectrum subtypes were only supported by prototypic case vignettes and not by standardized assessments.

Lewinshon et al. (1995) found that the prevalence of bipolar disorders in a representative community sample of 1,700 adolescents was approximately $1 \%$ and that an additional $5.7 \%$ of the sample reported having experienced a distinct period of abnormally and persistently elevated, expansive, or irritable mood even though they never met criteria for bipolar disorder. Compared with adolescents with a history of major depression or «never mentally ill», those with subthreshold bipolar symptoms showed significant functional impairment and high rates of comorbidity (particularly with anxiety and disruptive behavior disorders), suicide attempts, and mental health services utilization.

\section{CLINICAL IMPLICATIONS OF SPECTRUM APPROACH TO MOOD DISORDERS}

Under-diagnosis of subthreshold or atypical forms of mania may lengthen the time to identification of illness and this may delay treatment and worsen prognosis. Several factors contribute to the unrecognition and underdiagnosis of manic or hypomanic symptoms, compared to depressive symptoms (Cassano et al., 1999).

1. In contrast with a patient's experience of depression, mild manic symptoms are not usually associated with subjective distress, but rather with higher productivity, sociability, and expression of other attributes (i.e., swift thinking, assertiveness and attractiveness).

2. Individuals with manic symptoms are rarely seen in need of treatment, unless they have a prior history of severe mania, and are likely to resist treatment when it is offered or suggested. Self-perception of mild manic symptoms is usually ego-syntonic even when patient's cognitive and behavioral patterns have changed significantly.

3. Individuals with subthreshold manic symptoms refuse drug treatments with lithium or other mood-stabilizers and typically want psychotherapy. In psychotherapy settings, bipolarity may be overlooked because treatment focuses on psychodynamic conflicts or environmental stresses.

4. Subthreshold symptoms of mania may be interpreted as abnormal personality traits and, therefore, their connection with bipolar illness is often missed. In other cases, when subthreshold bipolar features co-occur with other psychiatric disorders, such as anxiety or eating disorders or alcohol abuse, the simultaneous presence of mood and behavioral instability and an anxious-phobic phenomenology can lead to a diagnosis of neurotic syndrome (Tyrer, 1989; 1990; Boulenger \& Lavallee, 1993).

5. Mania and hypomania in children and adolescents are often associated with attention deficit hyperactivity disorder or conduct disorders (Biederman et al., 1996; Milberger $e t$ al., 1995) and, usually, these latter diagnoses prevail (Kovacs \& Pollock, 1995; Lewhinson et al., 1995; Weller et al., 1995) or antisocial personality (Akiskal et al., 1995) (see table I). 
6. Cyclicity and seasonality in the phenomenology of the subthreshold bipolar symptoms means these symptoms may be overlooked. Conversely, the cyclicity itself may be a manifestation of bipolar spectrum. In particular, recurrence of periods of productivity, exuberance, irritability or arrogance should be carefully investigated. Similar subthreshold symptomatology may also occur during the various phases of the menstrual cycle, but these patterns may be frequently overlooked when they are not pronounced.

Table I. - Bipolar spectrum in special populations (adapted from Cas. sano et al., in press).

1. Bipolar spectrum in children and adolescents

2. Bipolar spectrum in the elderly

3. Bipolar spectrum in substance abusers

4. Bipolar spectrum in pregnancy

5. Bipolar spectrum and illegal behaviors

6. Bipolarity in mentally retarded or brain damaged

7. Bipolar spectrum associated with axis III medical conditions

These factors and the absence of operationalized criteria for subsyndromal forms of mania, may explain why the identification of numerous subthreshold expressions of mania may be difficult in clinical practice. The current international systems of classifications only identify a little range of prototypic symptoms to classify axis I and II disorders. In the absence of evident mood disregulations, recognition of several features (table II) such as, for instance, fluctuations of energy levels and alternating phases of psychomotor activity, as well as of neurovegetative functions and psychological drives should be considered fundamental indicators of subthreshold bipolar spectrum symptoms especially in young adults and children.

Table II. - Indicators of sub-syndromal bipolarity extrapolated from questions of the SCI-MOODS (adapted from Cassano et al., 1998).

1. Feel a strong desire to reconnect with people not seen or spoken with for a long time

2. Talkativeness, speaking rapidly and loudly

3. Sensation of never getting tired

4. Overactive and have such a high energy that is sometimes irritated or exhausted others

5. Pleasure in making jokes (even inappropriately)

6. Wearing clothing or hairstyle dramatic or extravagant, very high

fashion or unusual

7. Tend to behave in an opposite way

8. Frequent periods of abrupt shifts from one brief subthreshold bipolar phase to another

9. Feel as if others were causing all of your problems

10. Behave in an uninhibited way

11. Have diurnal or seasonal cyclic variations of any or all of the above

\section{THE STRUCTURED CLINICAL INTERVIEW FOR MOOD SPECTRUM (SCI-MOODS)}

The SCI-MOODS is a structured clinical interview designed to assess a wide variety of lifetime signs and symptoms belonging to the mood disorders spectrum. The SCIMOODS is intended to be comprehensive enough to detect current and lifetime, subthreshold and atypical manifestations not listed within the DSM criteria, and to determine whether they have a predictive clinical weight for determining treatment selection and outcome. The SCI-MOODS includes the classic DSM criterion symptoms for major depression and mania, as well as a wide range of subtle and atypical manifestations of mood disorders, not contemplated by DSM-IV, which could exist on their own, may occur in the context of a major depressive episode, may represent early onset precursors or prodromal symptoms, or residual symptoms that often interfere with overall functioning and quality of life. It is important to note that the SCI-MOODS does not enable the interviewer to make syndromal diagnoses. This can be accomplished only through formal diagnostic evaluation using either more traditional clinical interview methods or, preferably, through a structured clinical interview such as the SCID (Spitzer et al., 1996).

The interview underwent psychometric testing procedures that have been designed to assess its validity, stability and reliability (Fagiolini et al., 1999). In its present form it is composed of 140 items grouped into four domains: "Mood" (50 items), "Energy" (21 items), "Cognition" (46 items) and "Rhythmicity and Vegetative Functions" (23 items). The first three domains are divided into depressive and manic subdomains. The "Mood" domain is devoted to the subject's experience of mood lability and associated changes in interest in family, friends, romantic relationships, work, hobbies and sports. Questions about "Energy" record the presence of periods when energy levels change (see Appendix for specific questions included in this section of the interview). The "Cognition" domain explores changes in cognition associated with energy or mood fluctuations. "Rhythmicity and Vegetative Functions" are evaluated by exploring routine daily changes in mental and physical efficiency, mood changes, energy and physical well-being related to the weather, seasons, or the change of season, shifts in mood associated with changes in eating habits, sleep and sexual activities.

A detailed set of items in the SCI-MOODS aims to identify energy fluctuations, mood instability and emotional lability. This arrangement makes it possible to re- 
cognize and record the coexistence of conflicting phenomena such as excitement or hyperthymia with fatigue, thought acceleration with indecisiveness, hyper-stimulation with lack of productivity, anxiety-agitation with motor retardation and inhibition, mood elevation with irritability and hostility, and dysphoric mood with bursts of anger.

The systematic use of this structured interview should allow clinicians to define core symptoms and provides a method for identifying subthreshold signs of bipolarity, as well as of subthreshold symptoms of the unipolar mood spectrum.

In the absence of such a structured approach to the evaluation of the mood spectrum, the identification of subthreshold unipolar, as well as of bipolar, symptoms requires special clinical training and extensive clinical experience. With the carefully-constructed standardized diagnostic process that we are proposing with the SCIMOODS, identification of these phenomena by relatively inexperienced trainees becomes possible and the experience of conducting the interview becomes a form of training in this kind of sophisticated diagnostic work-up. The internal consistency of the instrument and its ability to discriminate between outpatients with unipolar disorder and patients with bipolar disorder and between two non-psychiatrically ill control groups has been recently assessed (Fagiolini et al., 1999). Inter-rater and test-retest reliability was also evaluated in a sample of subjects representative of the entire mood spectrum. The SCI-MOODS items were grouped a priori into four domains, three of which were then divided into depressive and manic subdomains. The analyses conducted demonstrate that these constructs are psychometrically sound. Each domain has good internal consistency and the correlation between them is positive and high, suggesting that it makes sense to consider these domains as aspects of a unitary phenomenon, mood spectrum. The correlations among the three manic subdomains and among the three depressive subdomains were consistently higher than those between manic and depressive subdomains, denoting good construct validity.

As expected, unipolar and bipolar patients had consistently higher mean scores on all subdomains and domains than non-psychiatric control groups. Bipolar patients displayed the highest scores on manic subdomains but they did not differ from unipolar patients in the mean scores of depressive subdomains. Results of the discriminant analysis showed that the "Energy" domain, the "Cognition" manic subdomain and the "Mood" depressive subdomain were particularly useful in discriminating the subjects with bipolar disorder from those with unipolar disorder. Over $80 \%$ of cases were correctly classified using only questions from these three sections of the interview. The inter-rater and test-retest reliability of the SCI-MOODS was found to be excellent. These results provide initial empirical support for the utility of the SCI-MOODS, suggesting that it may be an accurate screening instrument for mood spectrum psychopathology, not only in psychiatric settings, but also in the general health care sector and in the general population.

\section{CONCLUSION}

The insufficiency of threshold psychiatry is clearly demonstrated by the frequency and clinical relevance of the so-called 'subthreshold depressive and bipolar syndromes'. Although data from epidemiological literature are in general consistent in showing the relatively more favorable outcome of minor mental disorders as compared to threshold ones, a considerable proportion of cases with subthreshold forms of depression may have a negative outcome or a tendency to transit into a threshold mental disorder disorders (Tiemens et al., 1996; Pini et al., 1999).

However, there seems to be a clear tendency by physicians to detect patients with more severe psychiatric illness, while minor psychiatric conditions tend to remain unrecognized and unmanaged (Ormel et al., 1996; Coyne et al., 1995; Pini et al., 1997).

In psychiatric settings, depressive symptoms, though of low severity, are easily recognized as such. However, these subthreshold syndromes are, at best, defined in a negative light, i.e. as cases not meeting the threshold for any DSM-IV or ICD-10 diagnosis; they should, instead, be defined 'positively' on the basis of the subtle symptomatology, clinical course and functional impairment that they may produce.

Subthreshold and enduring bipolar symptomatology, with an onset in childhood or adolescence, turned out to be linked with subsequent, later-onset major episodes so that the hypothesis which has been proposed represents the subtle symptomatology as the basic nuclear core that, by interacting with the environment, generates the fullblown disorder (Akiskal, 1983). According to this view, the disease is initially expressed at a "temperamental" level or, more precisely, as a subthreshold symptom pattern.

The findings described in this review should be also interpreted in the light of recent debate and a re-appraisal of the importance of mental illness in non-psychia-

Epidemiologia e Psichiatria Sociale, 9, 3, 2000 
tric settings. It has been argued that minor mental disorders seen in primary care have high likelihood of spontaneous recovery (Goldberg, 1992; Katon et al., 1994). Some recent studies suggest that outcomes of mental disorders in primary care are not simply related to their initial severity and that recognition by the physician is not always associated with a better outcome (Pini $e t$ al., 1999). Future research should clarify what psychopathological conditions deserve attention and treatment.

Up to date greater attention has been focused on subthreshold features of depression and a large amount of clinical and epidemiological data have been collected in this area which represents a limited fraction of mood disorders. Scarce data are available on the other wide area of mood spectrum represented by manic and mixed subthreshold manifestations of bipolar disorder. The spectrum model for mood disorders, as it has been proposed by a group of clinical investigators (Cassano et al. 1997; 1999), aims at a comprehensive descriptive and diagnostic approach which may allow a simultaneous assessment of unipolar and bipolar subthreshold symptoms. The systematic assessment of mood spectrum was made possible by designing the SCI-MOODS, which is now available, both as interviewer-administered version and self-report questionnaire, for clinical, research and educational purposes.

\section{REFERENCES}

Akiskal H.S. (1983).The bipolar spectrum: new concepts in classification and diagnosis. In Psychiatry Update, Vol. II (ed. C. Grinspoon), pp. 271-292. American Psychiatric Press: Washington DC.

Akiskal H.S. \& Pinto O. (1999). The evolving bipolar spectrum: prototypes I, II, III and IV. Psychiatric Clinics of North America 22, 517-534.

Akiskal H.S., Rosenthal T.L., Haykal R.F., Lemmi H., Rosenthal R.H. \& Scott-Strauss A. (1980). Characterological depressions: clinical and sleep EEG findings separating "subaffective dysthymias" from "character spectrum disorders". Archives of General Psychiatry 37 , $777-783$.

Akiskal H.S., Maser J.D., Zeller P.J., Endicott J., Coryell W., Keller M., Warshaw M., Clayton P. \& Goodwin F. (1995). Switching from 'unipolar' to bipolar II: a 11 year prospective study of clinical and temperamental predictors in 559 patients. Archives of General Psychiatry 52, 114-123.

Angst J. \& Merikangas K. (1997) The depressive spectrum: diagnostic classification and course. Journal of Affective Disorders 45 , $31-40$.

Biederman J., Faraone S., Mick E., Wozniak J., Chen L., Ouellette C., Marrs A., Moore P., Garcia J., Mennin D. \& Lelon. E. (1996). Attention-Deficit Hyperactivity Disorder and Juvenile Mania: an overlooked comorbidity? Journal of American Academy of Child Adolescence Psychiatry 35, 997-1008.

Boulenger J.P. \& Lavallee Y.J. (1993). Mixed anxiety and depression:
Diagnostic issues. Journal of Clinical Psychiatry 54, Suppl. 1, 3-8. Cassano G.B. \& Savino M. (1993). Chronic major depressive episode and dysthymia: comparison of demographic and clinical characteristics. European Psychiatry 8, 277-279.

Cassano G.B. \& Savino M. (1997).Chronic and residual major depressions. In Dysthymia and the Spectrum of Chronic Depressions (ed. H.S. Akiskal and G.B. Cassano G.B.), pp. 54-65. Guilford Press: New York.

Cassano G.B., Maggini C. \& Akiskal H.S. (1983). Short-term subchronic and chronic sequelae of affective disorders. Psychiatric Clinics of North America 6, 55-68.

Cassano G.B., Michelini S., Shear M.K., Coli E., Maser J.D. \& Frank E. (1997). The panic-agoraphobic spectrum: a descriptive approach to the assessment and treatment of subtle symptoms. American Journal of Psychiatry 154, Suppl. 6, 27-38.

Cassano G.B., Dell'Osso L., Frank E., Miniati M., Fagiolini A., Shear K., Pini S. \& Maser J.D. (1999). The bipolar spectrum: a clinical reality in search of diagnostic criteria and an assessment methodology. Journal of Affective Disorders 54, 319-328.

Cassano G.B., McElroy S., Brady K., Nolen W. \& Placidi G.F. (in press). Current issues in the identification and management of bipolar spectrum disorders in 'special populations'. Journal of Affective Disorders.

Coyne J.C., Schwenk T.L. \& Fechner-Bates S. (1995). Nondetection of depression by primary care physicians reconsidered. General Hospital Psychiatry 17, 3-12.

Fagiolini A., Dell'Osso L., Pini S., Armani A., Bouanani S., Rucci P., Cassano G.B., Endicott J., Maser J.D., Shear M.K., Grochocinski V. \& Frank E. (1999). Validity and reliability of a new instruments for assessing mood symptomatology: the Structured Clinical Interview for Mood Spectrum (SCI-MOODS). Journal of Methods in Psychiatric Research 8, 71-82

Frank E., Cassano G.B., Shear K., Rotondo A., Dell'Osso L., Mauri M., Maser J.D. \& Grochocinski V. (1998). The spectrum model: a more coherent approach to the complexity of psychiatric symptomatology. CNS Spectrums 4, 23-34.

Goldberg D. (1992) The treatment of mental disorders in general medical settings. General Hospital Psychiatry 14, 83-85.

Hollander E. \& Wong C.M. (1995). Obsessive-compulsive spectrum disorders. Journal of Clinical Psychiatry 56, 3S-5S.

Judd L.L. \& Akiskal H.S. (2000). Delineating the longitudinal structure of depressive illness: beyond clinical subtypes and duration thresholds. Pharmacopsychiatry 33, 3-7.

Judd L.L., Rapaport M.H., Paulus M.P., Brown J.L. (1994). Subsyndromal symptomatic depression: a new mood disorder? Journal of Clinical Psychiatry 55, 18S, 28S.

Katon W., Lin E., VonKorff M., Bush T., Walker E., Simon G. \& Robinson P. (1994). The predictors of persistence of depression in primary care. Journal of Affective Disorders 31, 81-90.

Kessler R.C. (1997). The prevalence of psychiatric comorbidity. In Treatment Strategies for Patients with Psychiatric Comorbidity (ed. S. Wetzler and W.C. Sanderson), pp. 23-48. Wiley \& Sons: New York.

Kety S.S. (1987). The significance of genetic factors in the etiology of schizophrenia: results from the national study of adoptees in Denmark. Journal of Psychiatric Research 21, 423-429.

Kovacs M. \& Pollock M. (1995). Bipolar disorder and comorbid conduct disorder in childhood and adolescence. Journal of American Academy of Child Adolescence Psychiatry 34, 715-723.

Lewinsohn P.M., Klein D.N. \& Seeley J.R. (1995). Bipolar disorders in a community sample of older adolescents: prevalence, pheno-

Epidemiologia e Psichiatria Sociale, 9, 3, 2000 
menology, comorbidity, and course. Journal of American Academy of Child Adolescence Psychiatry 34, 454-63

Milberger S., Biederman J., Faraone S.V., Murphy J. \& Tsuang M.T. (1995). Attention Deficit Hyperactivity Disorder and Comorbid Disorders: issues of overlapping symptoms. American Journal of Psychiatry 152, 1793-1799.

Olfson M., Broadhead W.E., Weissman M.M., Leon A.C., Fraber L., Hoven C. \& Kathol R.(1996). Subthreshold psychiatric symptoms in a primary care group practice. Archives of General Psychiatry $53,880-886$.

Pini S., Cassano G.B., Simonini E., Savino M., Russo A. \& Montgomery S.A. (1997). Anxiety disorders comorbidity in unipolar depression, bipolar depression and dysthymia. Journal of Affective Disorders 42, 145-153.

Pini S., Berardi D., Rucci P., Piccinelli M., Neri C., Tansella M. \& Ferrari G. (1997). Identification of psychiatric distress by primary care physician. General Hospital Psychiatry 19, 411-418.

Pini S., Perkonnig A., Tansella M. \& Wittchen H-U. (1999). Prevalence and 12-month outcome of threshold and subthreshold mental disorders in primary care. Journal of Affective Disorders 56, 37 48.

Rapaport M. \& Judd L. (1998). Minor depressive disorder and subsyndromal depressive symptoms: functional impairment and response to treatment. Journal of Affective Disorders 48, 227-232.

Schotte K. \& Cooper B. (1999). Subthreshold affective disorders: a useful concept in psychiatric epidemiology. Epidemiologia e Psichiatria Sociale 8, 255-261.

Spitzer R.L., Williams J.B.W., Gibbon M. \& First M.B. (1996). Structured Clinical Interview for DSM-IV - SCID-I. New York State Psychiatric Institute, Biometrics Research: New York.

Sullivan P.F., Kessler R.C. \& Kendler K.S. (1998). Latent class analysis of lifetime depressive symptoms in the national comorbidity survey. American Journal of Psychiatry 155, 1398-1406.

Tiemens B.G., Ormel J. \& Simon G. (1996). Occurrence, recognition and outcome of psychological disorders in primary care. American Journal of Psychiatry 153, 636-644.

Tyrer P. (1989). Classification of Neurosis. John Wiley \& Sons: New York.

Tyrer P.J. (1990). The division of neurosis: a failed classification. Journal of Royal Society of Medicine 83, 614-616.

Van Valkenburg C., Akiskal HS, Puzantian V. \& Rosenthal T. (1994). Anxious depression: clinical, family history, and naturalistic outcome-comparison with panic and major depression disorders. Journal of Affective Disorders 6, 67-82.

Weller E., Weller R. \& Fristad M. (1995). Bipolar disorder in children: misdiagnosis, underdiagnosis, and future directions. Journal of American Academy of Child Adolescence Psychiatry 34, 709. 714.

Winokur G. (1974). Duration of illness prior to hospitalization (onset) in the affective disorders. Neuropsychobiology 2, 87.93. 